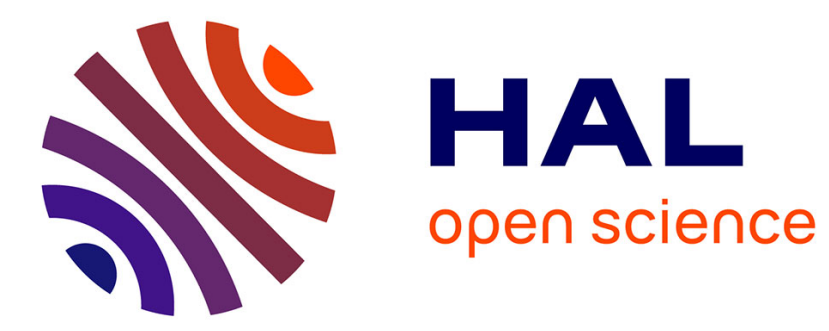

\title{
Technology assessment for radio communication between UAV and Ground: qualitative study and applications
}

\author{
Najett Neji, Tumader Mostfa, Yasmina Bestaoui
}

\section{To cite this version:}

Najett Neji, Tumader Mostfa, Yasmina Bestaoui. Technology assessment for radio communication between UAV and Ground: qualitative study and applications. 89th IEEE Vehicular Technology Conference (VTC2019-Spring), Apr 2019, Kuala Lumpur, Malaysia. hal-02062408

\section{HAL Id: hal-02062408 https://hal.science/hal-02062408}

Submitted on 8 Mar 2019

HAL is a multi-disciplinary open access archive for the deposit and dissemination of scientific research documents, whether they are published or not. The documents may come from teaching and research institutions in France or abroad, or from public or private research centers.
L'archive ouverte pluridisciplinaire HAL, est destinée au dépôt et à la diffusion de documents scientifiques de niveau recherche, publiés ou non, émanant des établissements d'enseignement et de recherche français ou étrangers, des laboratoires publics ou privés. 


\title{
Technology assessment for radio communication between UAV and Ground: qualitative study and applications
}

\author{
Najett Neji, Tumader Mostfa, and Yasmina Bestaoui Sebbane
}

\begin{abstract}
In this paper, we develop an analytic methodology to determine the best technology to carry the communication between an Unmanned Aerial Vehicle (UAV) and a ground control station (GCS). We assume herein that the UAV is performing its mission under nominal conditions. For this, we identify some relevant criteria that cover most use-cases. We propose a multi-criteria analysis to determine the best technology to carry the radiocommunication between the UAV and the GCS. In this work, we distinguish between the Control and Non-Payload Communication Channel (CNPC) and the Payload Channel. By studying two different missions, we emphasize that the technology assessment results depend on the use-case as well as the UAV scenario, and that for a same scenario the results for CNPC are different from the Payload communication. In this work, we are focused on the precise agriculture (PA) use-case, and the public safety (PS) use case. We present the assessment results in both Visual Line of Sight (VLOS), and Beyond Line of Sight (BVLOS) scenarios. The latter is very interesting because the communication UAV - GCS becomes of critical importance.
\end{abstract}

\section{INTRODUCTION AND RELATED WORKS}

Owing to their compact size, their reduced weight and increasing capabilities, Unmanned Aerial Vehicles (UAV) are nowadays used in a wide range of civil applications. Three typical categories of missions are identified [1]: UAV-aided ubiquitous coverage (e.g. in case of infrastructure damage and crowded areas), UAV-aided relaying (e.g. between frontline and command center for emergency responses), and UAV-aided information dissemination and data collection (e.g. for precision agriculture). To accomplish its mission, the UAV exchanges information with a Ground Control Station (GCS), through two communication channels: The control and non-payload communication (CNPC) channel and the payload channel. Both of them characterize the Air-to-Ground (AG) communication.

The CNPC is responsible for ensuring safe, reliable, and effective UA flight operation. The World Radiocommunication Conference (WRC'12) allocated C-Band (5030-5091 MHz) and L-Band (960-977MHz) to CPNC. The payload link is responsible for transmitting data (photos, videos...) between both components. This channel requires less security than CNPC, and its uses unlicensed Industrial Scientific and Medical bands 2.4 $\mathrm{GHz}$ and $5.8 \mathrm{GHz}$, also known as Wi-Fi bands.

According to the distance separating the UAV and the base station, the regulation instances defined three main scenarios:
VLOS (Visual Line of Sight) and EVLOS (Extended Line of Sight), where the UAV is kept UAV in visual-line-of-sight at all times, and BVLOS (Beyond Line of Sight), where the pilot has no visual reference of the UAV.

While UAVs are used worldwide in (E)VLOS scenarios, their usage for BVLOS scenarios is still challenging, to introduce UAVs into the National Air Space (NAS). Numerous researches deal with the BVLOS, because the communication between the $\mathrm{UAV}$ and the GCS becomes of crucial importance. In addition, commonly used economical wireless modules are restricted by the short communication range and are easily blocked by obstacles in BVLOS [2].

In this paper, we are interested in the radio communication between the UAV and the GCS. This field is of interest for UAVs, as mentioned in [3]. To the best of our knowledge, for both use cases, the communication architecture is implemented within the UAV and is kept invariant the whole duration of the mission. Each mission/use case has its own constraints and requirements, and the most suitable technology for $\mathrm{AG}$ communication depends on the application. The majority of deployed UAV in the market use IEEE 802.11 technologies (WiFi) or IEEE 802.15.1 (Bluetooth) for both CNPC and payload links. Several research projects highlighted the benefits of using other technologies for AG communications: The SHERPA Project ("Smart Collaboration between Humans and groundaErial Robots for imProving rescuing activities in Alpine environment"), which proved theoretically the utility of Wimax for safety-oriented application [4]. In addition, the IEEE 802.15.4 (Zigbee) and Low Power Wide Area Networks (LPWAN) standards have been identified as the most suitable for precision agriculture scenarios [5].

Based on this context, we propose in this paper an analytical approach to determine which technology fits best for the communication between the UAV and GCS radios. The paper is organized as follows. In section II, we present the overview of our proposed methodology for technology assessment. We apply it for both CNPC link and payload link, in section III and section IV, respectively. For each link, we are interested in two usecases: The Precise Agriculture and the Public Safety, and in two scenarios: the VLOS and the BVLOS. Section V is dedicated to analysis of the results and discussions. Finally, we present in section VI our main conclusions and our ideas for further work. 


\section{TECHNOLOGY ASSESSMENT METHODOLOGY: OVERVIEW}

In this section, we present our analytical approach to assess the existing and emerging technologies for AG communications.

This approach is inspired from technology assessment works that were developed by the aeronautical community between 2004 and 2007, in the context of developing the aeronautical future communication system (FCS) under the AP-17 project [6]. It should be noticed that the findings of the FCS are not directly applicable because of different requirements between a UAV to GCS link and a general aeronautical data link [7].

Our approach is based on the following steps:

\section{A. Select the candidate technologies}

After a first technology screening, we decided to investigate technologies which operate, or are expected to operate in the frequency bands used by UAVs. Our shortlist is composed by:

- Technologies from the Wireless Local Area Networks (WLAN) family: this type of networks can provide high data rates and acceptable communication range. Herein we focus on the IEEE 802.11 technology known as Wireless Fidelity (Wi-Fi).

- Technologies from the Wireless Metropolitan Area Networks (WMAN) family: this type of networks can provide larger communication ranges, but lower data rates than WLAN. We investigate the IEEE 802.16 standard, known as Worldwide Interoperability for Microwave Access (Wimax).

- Technologies from the Wireless Personal Area Networks (WPAN) family: these networks are characterized by short range and low energy consumption. We analyze the IEEE 802.15.1 (리 (Zigbee).

- Technologies from the Wireless Wide Area Networks (WWAN) family: also named cellular networks, they can provide both very high data rates and large coverage but they are characterized by high energy consumption. We focus on the Unlicensed Long Term Evolution (LTE-U) [8], and on the next generation of cellular networks $(\underline{5 \mathrm{G}})$. LTE-U initiative by Qualcomm has led to a standard for operating LTE-A over the unlicensed spectrum. 5G standardization will be finalized in 2020 .

- Technologies from the Low Power Wide Area Networks (LPWAN) family: these networks have large communication ranges and low energy consumption. We analyze SigFox, M2M Ingenu and Lora technologies. SigFox [9] is the first LPWAN technology proposed in the Internet of Things market, and was founded in 2009. M2M Ingenu is an emerging star in the landscape of LPWANs, developed by On-Ramp Wireless (in California). Lora is a new LPWAN solution, designed and patented by Semtech Corporation.

\section{B. Identify the criteria}

In this step, we define the criteria that will help us to determine the most suitable technology. We identify 9 criteria:

- Communication range: we compare the technology maximum range and the needed distance between the pilot and the UAV to perform the mission.

- Energy consumption: we notice the lower is the energy consumption, the longer is the battery lifetime, the UAV would be able to achieve missions at larger distances.

- Cost: we mainly consider the ground infrastructure cost, because spectrum is free (payload) or allocated (CNPC) and equipment cost would be similar, irrespective of the technology.

- $\quad$ Throughput: we compare the provided data rate by the technologies based on their specifications and the required throughput by the application.

- Robustness: we investigate the technology immunity against external noise and interference from other radio systems in the surroundings.

- Security: a particular interest is put on technology immunity against hacking. This criterion is important in BVLOS scenario because the pilot has to rely on data provided by the UAV which is not in visual line of sight.

- Latency: in applications where real time transmission is needed, it is important to take into account the time duration between information sending by the UAV and its treatment by the ground station.

- Technology maturity: we refer to the Technological Readiness Level (TRL), ranging from 1 (basic research) to 9 (fully deployed). We evaluate the TRL based on technologies deployment in the UAV market.

- Interoperability with other standards: we take into account the trend for heterogeneous networks, considering that a UAV using a technology which is able to exchange information with other standards is more likely to perform its mission in better conditions.

\section{Define the scenario}

In this step, we determine three parameters: the use-case, the UAV scenario and the AG link. The use case is related to the application (e.g. precise agriculture, public safety, packet delivery...). The UAV scenario is either (E)VLOS or BVLOS. The AG link is either CNPC link or payload link.

\section{Classify the criteria}

In this step, we classify the previously mentioned criteria into two categories: the essential criteria and the desirable criteria. The essential criteria are the most challenging factors according to the state-of-the-art. The desirable criteria are the other ones. 


\section{E. Evaluate the suitability between criteria and technologies}

In this step, we analyze if each technology is likely to fulfill each of the criteria. For this, we assess the technologies based on the scores given in Table I for essential criteria, and in Table II for desirable criteria.

we consider that the essential criteria are the 3 most critical ones, with respect to the application requirements. Hence, for each assessment, there will be 3 essential criteria and 6 desirable criteria.

In addition, we scored the criteria as follows. If the technology meets an essential criterion, it is scored 10, else it is scored 0 . For desirable criteria, the scores are 3 (high suitability), 2 (reasonable suitability), and 1 (low suitability). Using such a scoring, a technology which does not meet an essential criterion is not likely to be the best technology, even if it meets several desirable criteria.

TABLE I. TECHNOLOGY EVALUATION FOR ESSENTIAL CRITERIA

\begin{tabular}{|l|l|}
\hline \multicolumn{1}{|c|}{ Assessment level } & \multicolumn{1}{c|}{ Signification } \\
\hline 10 & Meets the requirement \\
\hline 0 & Does not meet the requirement \\
\hline
\end{tabular}

TABLE II. TECHNOLOGY EVALUATION FOR DESIRABLE CRITERIA

\begin{tabular}{|l|l|}
\hline \multicolumn{1}{|c|}{ Assessment level } & \multicolumn{1}{c|}{ Signification } \\
\hline 3 & High suitability \\
\hline 2 & Acceptable suitability \\
\hline 1 & Low suitability \\
\hline
\end{tabular}

\section{F. Technology scoring}

In this step, we determine, for each technology, the global score, by adding the different assessment scores. We consider that the best technology is the one having the highest score.

\section{TECHNOLOGY ASSESSMENT FOR CNPC LINK}

In this section, we intend to apply our methodology for the CNPC link. We recall that the CNPC link is responsible for ensuring safe, reliable, and effective UA flight operation. These requirements are independent of the type of the mission. As a consequence, we consider that the essential criteria cover the communication range, the throughput and the security.

The other criteria are considered as desirable criteria. To compare the technologies against essential criteria, we take into account information from the literature. According to [10], future CNPC data link(s) must be able to accommodate up to $320 \mathrm{kbps}$ per UA. For the communication range, and based on the European UAV regulation, the VLOS scenario is limited to a distance of 500 meters between the UAV and the GCS.

- communication range: the technology meets requirements it provides a larger range than the distance between devices.
- throughput: the technology meets the requirements if its throughput is at least $320 \mathrm{kbps}$.

- security: the technology security is satisfying for the link if it uses Advanced Encryption Standard (AES).

Then we provide our assessment for the desirable criteria:

- energy consumption: we consider that the technology is suitable if its energy consumption is low. Medium energy consumption is acceptable.

- cost: we considered that a technology with low costs fulfills the application requirements. Medium cost is acceptable.

- robustness: based on the literature, we investigate the bit error rate (BER) with respect to the signal to noise ratio (SNR). This metric is related to the used signal modulation. We extract this information from technology specifications.

- latency: we consider that the technology is suitable if its latency is low, which means that real time transmission is possible. Medium latency is acceptable.

- technology maturity: If the TRL is $1-2-3$, we attribute the lowest score. If the TRL is 4-5-6, the maturity is acceptable. If the TRL is 7-8-9, the technology is highly suitable.

- interoperability with other standards: we attribute the highest score for technologies which are interoperable with the other standards. If the interoperability issue is under progress, we attribute the medium score. Otherwise, we attribute the lowest.

\section{A. VLOS scenario}

We provide in Table III the technology assessment results for the CNPC link. For each technology, we assign 0 or 10 for the essential criteria and 1 or 2 or 3 for the desirable criteria. We detailed in a recent work [11] the justification of each attributed score, based on technology specifications, as well as recent research works and information from products in the market.

Using results from Table III, we summarize in Fig. 1 the results for this scenario. We presented histograms giving the total score of each technology. We can see that for the CNPC, LTE-U and Wimax technologies are good candidates. This is coherent with the literature, namely the technology assessment made by NASA in 2014 [7]. We can also notice that 5G seems to be the best candidate, owing to optimistic assessment in terms of security and throughput. 


\begin{tabular}{|c|c|c|c|c|c|c|c|c|c|}
\hline technology & $\underline{\text { wifi }}$ & wimax & bluetooth & zigbee & LTE UN & Lora & Sigfox & M2M INGENU & $\underline{\underline{5 G}}$ \\
\hline \multicolumn{10}{|l|}{ Essential Criteria } \\
\hline communication range & 0 & 10 & 0 & 10 & 10 & 10 & 10 & 10 & 10 \\
\hline security & 0 & 10 & 0 & 10 & 10 & 10 & 0 & 10 & 10 \\
\hline throughput & 10 & 10 & 10 & 0 & 10 & 0 & 0 & 0 & 10 \\
\hline \multicolumn{10}{|l|}{ Desirable criteria } \\
\hline energy consumption & 1 & 1 & 2 & 3 & 1 & 3 & 3 & 3 & 3 \\
\hline cost & 2 & 1 & 3 & 3 & 1 & 1 & 1 & 2 & 1 \\
\hline robustness & 2 & 1 & 2 & 3 & 1 & 2 & 3 & 2 & 3 \\
\hline latency & 1 & 2 & 1 & 2 & 2 & 2 & 1 & 1 & 3 \\
\hline technology maturity & 3 & 1 & 3 & 2 & 2 & 1 & 1 & 1 & 1 \\
\hline interoperability & 3 & 3 & 2 & 3 & 2 & 1 & 1 & 1 & 2 \\
\hline SCORE & 22 & 39 & 23 & 36 & 39 & 30 & 20 & 30 & 43 \\
\hline
\end{tabular}

TABLE III: TECHNOLOGY ASSESSMENT FOR THE CNPC LINK, VLOS SCENARIO

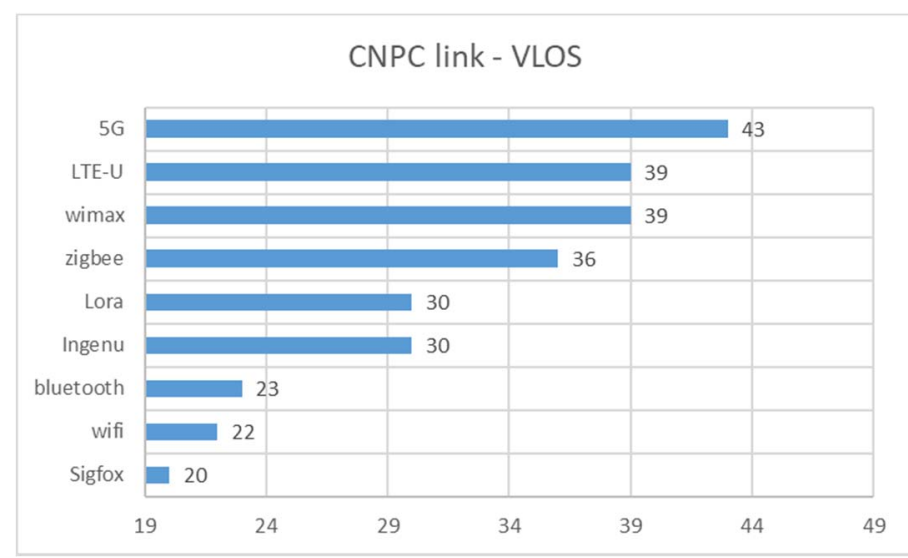

Figure 1: Assessment results for CNPC VLOS scenario

\section{B. BVLOS scenario}

We depict in Fig.2 the results for the CNPC BVLOS scenario. The results are similar to the previous case. The only exception is that the Zigbee no longer meets the requirements in terms of communication range.

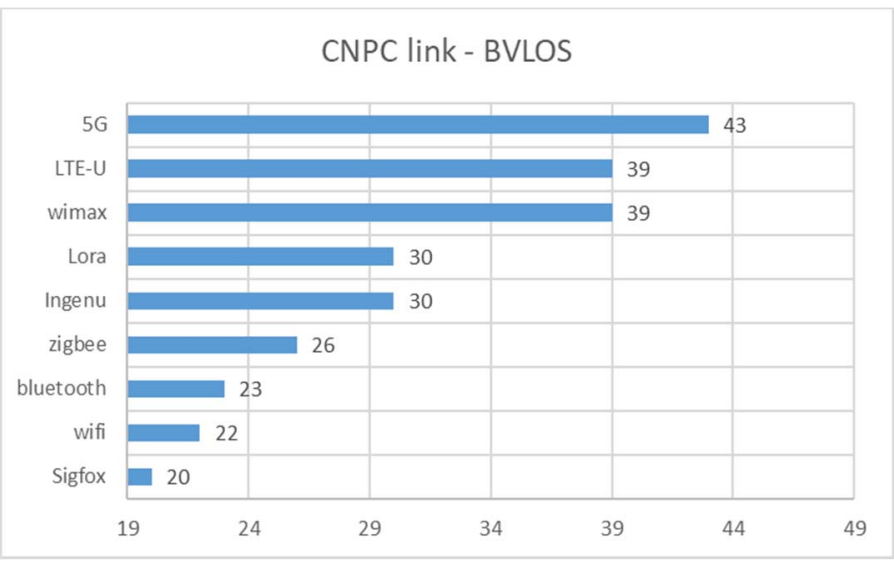

Figure 2: Assessment results for CNPC BVLOS scenario

\section{TECHNOLOGY ASSESSMENT FOR PAYLOAD LINK}

In this section, we intend to apply our methodology for the payload link. We recall that the payload link is responsible for transmitting data and voice between the flying equipment and the ground equipment. Hence, the requirements highly depend on the type of the mission. As a consequence, before classifying the criteria (essential vs. desirable), we need to have better insights about the specific requirements and constraints of the application. For this reason, we investigate in the two following subsections different use-cases: The Precise Agriculture (PA) and the Public Safety (PS).

\section{A. Precise agriculture use-case}

The precision agriculture (PA) scenario, also known as satellite farming or site specific crop management. The idea is to optimally manage farming based on observing, measuring and responding to inter and intra-field variability in crops.

Based on recent published works, the most challenging items for UAV application to PA are the communication range, the cost and the energy consumption [5]. We classify the criteria as follows: the essential criteria are the communication range, the cost and the energy consumption. The desirable criteria are the other ones.

\section{1) PA VLOS scenario}

We illustrate in Fig.3 the results for the PA Payload link VLOS scenario. We can see that Zigbee is the best candidate for this scenario because it meets the requirements in terms of cost, energy consumption and communication range. We also notice that M2M technologies are good candidates, and this is similar to results from literature.

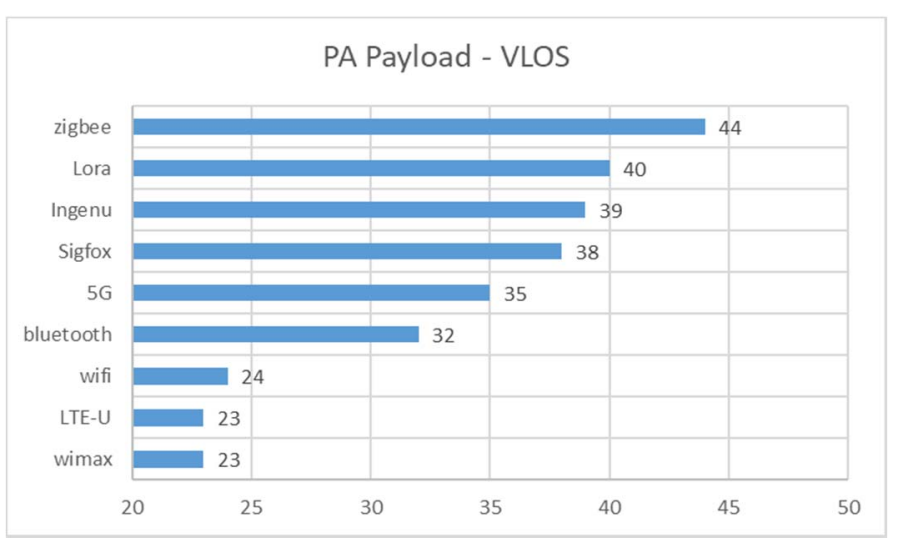

Figure 3: Assessment results for PA Payload VLOS scenario 


\section{2) PA BVLOS scenario}

We depict in Fig.4 the results for the PA Payload link VLOS scenario. The results are similar to the previous case. There are two exceptions. The first is that the Zigbee no longer meets the requirements in terms of communication range. The second is that security becomes an essential criterion.

Wi-Fi, Sigfox and Bluetooth do not satisfy the security requirements. Hence, we update score from Table III to obtain the results in Fig.4.

\section{B. Public safety use-case}

UAVs become nowadays a precious aid for public safety. For example, when an incident occurs, PS actors need to exchange precise and fast information to quickly take decisions for search and rescue. Information recorded by UAV are important and must be reliable.

Based on the literature, the essential criteria are the interoperability with other standards, the throughput and the security. The other criteria are desirable. For the technology assessment, we adopt the same approach as in the PA case. We also distinguish between the VLOS and BVLOS scenarios.

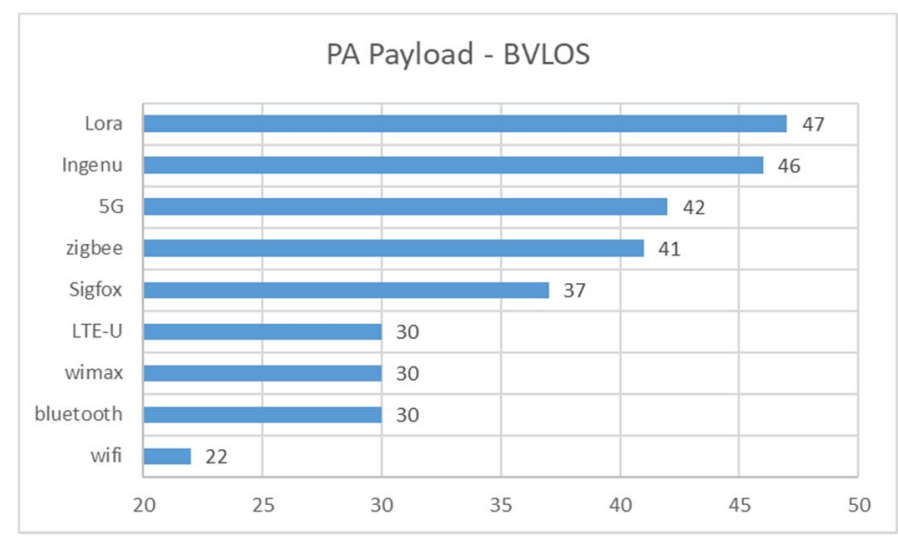

Figure 4: Assessment results for PA Payload BVLOS scenario

\section{1) VLOS scenario}

We provide in Fig. 5 the results for the PS Payload link VLOS scenario. We can see that LTE-U and Wimax are good candidates because they meet the requirements in terms of essential criteria and this is coherent with the state-of-the-art [12]. We can also notice that $5 \mathrm{G}$ seems to be the best candidate (same assumptions as previously).

\section{2) BVLOS scenario}

We provide in Fig.6 the results for the PS Payload link BVLOS scenario. The only exception compared to the VLOS scenario is that Zigbee does not satisfy the communication range requirements.

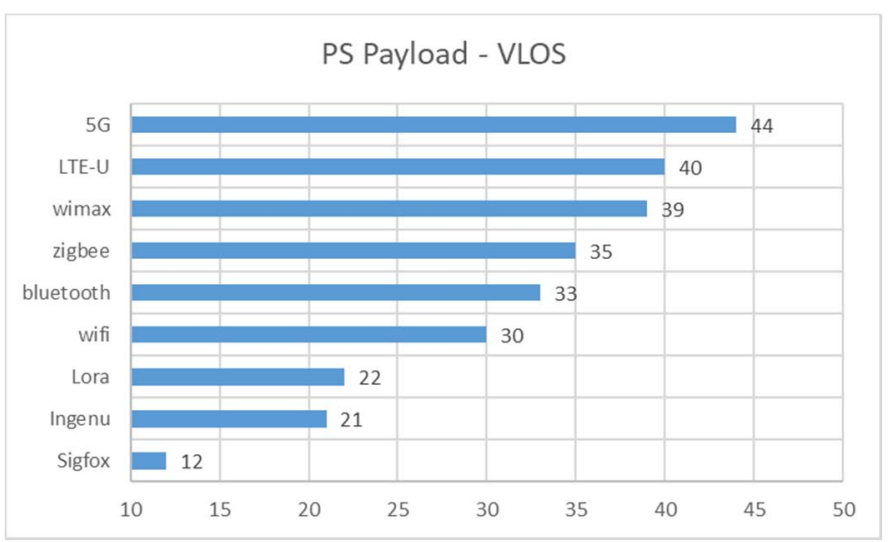

Figure 5: Assessment results for PS Payload VLOS scenario

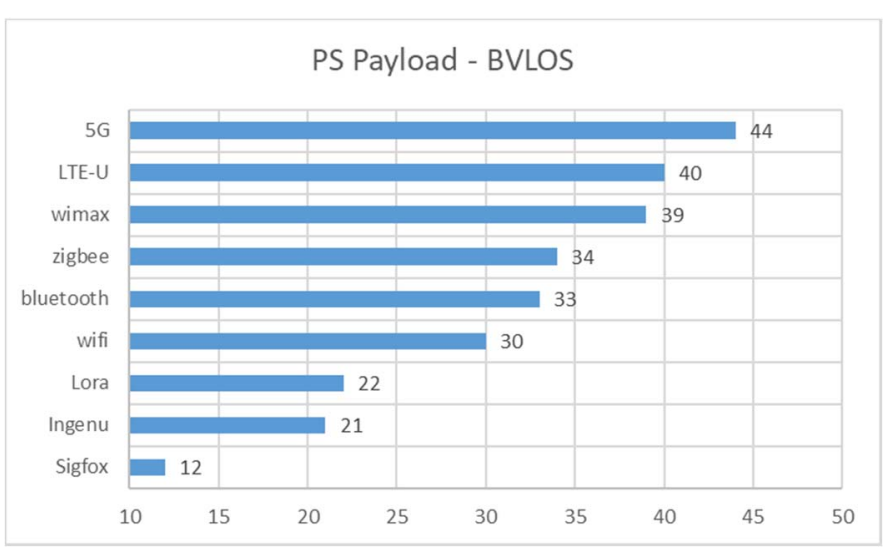

Figure 6: Assessment results for PS Payload BVLOS scenario

\section{DISCUSSION}

\section{A. Main results}

From these figures, we can first mention that the Wi-Fi technology scores are low compared to other technologies, for both studied scenarios, for CNPC and Payload links. Indeed, the Wi-Fi has been very attractive so far for AG communication, as it provides very high throughputs, enabling very-low latency missions. However, the Wi-Fi is known to be high-energy consuming, whereas one of the main UAV challenges today is to preserve the battery such that the UAV could perform longer missions. In addition, our results are coherent with the state-ofthe-art for CNPC assessment and Payload assessment in both PA and PS scenarios. Finally, we can notice that CNPC assessment is independent of the type of the mission, whereas the Payload assessment is task specific.

\section{B. Discussion}

Within the technology assessment, we expected that the $5 \mathrm{G}$ will provide good performance like low power consumption and high security levels. Based on promising researches on its capabilities, we attributed optimistic scores to 5G. In addition, LPWAN technologies scores are likely to increase in the near future, they are still under development. 


\section{CONCLUSION AND FURTHER WORK}

In this paper, we presented an analytical approach enabling technology assessment, to optimize the communication between an UAV and a GCS in nominal conditions. We used a multicriteria analysis to determine theoretically the most suitable technology, depending on the link nature (CNPC, Payload), usecase (PA, PS), and scenario referenced to current UAV regulation (VLOS, BVLOS). Our results are coherent with the literature and emphasize that the CNPC and Payload assessment are different. Further work will be focused on implementing an artificial intelligence layer within the UAV to dynamically change its radio communication technology with the GCS based on information from the environment and energy efficiency, and assuming that CNPC and Payload do not necessarily use the same technology.

\section{REFERENCES}

[1] Y. Zeng et al, "Energy-Efficient UAV Communication with Trajectory Optimization", IEEE Transactions on wireless communications, 2017, Vol 16, Issue 6, pp. $3747-3760$.

[2] Li, B.; Jiang, Y.; Sun, J.; Cai, L.; and Wen, C.-Y, "Development and Testing of a Two-UAV Communication Relay System", MDPI Sensors, 13 October 2016.

[3] M. Mozaffari, W. Saad, M. Bennis, Y. Nam, and M. Debbah, "A tutorial on UAVs for wireless networks: Applications, challenges, and open problems," in arXiv:1803.00680, March 2018.

[4] M. A. Rahman, "Enabling drone communications with WiMAX technology," in Proc. The 5th International Conference on Information, Intelligence, Systems and Applications (IISA), 2014.

[5] Jawad HM, Nordin R, Gharghan SK, Jawad AM, Ismail M., "EnergyEfficient Wireless Sensor Networks for Precision Agriculture: A Review", MDPI Sensors, 3 August 2017.

[6] FAA and Eurocontrol, "Action Plan 17: Future Communications Study, Final Conclusions and Recommendations, version 1.1, November 2017, https://www.eurocontrol.int/articles/future-communication-infrastructuresesar-activities.

[7] National Aeronautics and Space Administration, NASA STI program, "Communications Technology Assessment for the Unmanned Aircraft System (UAS) Control and Non-Payload Communications (CNPC) Link", NASA/CR-2014-216675, June 2014.

[8] Mina Labib, Vuk Marojevic, Jeffrey H. Reed, and Amir I. Zaghloul, "Extending LTE into the Unlicensed Spectrum: Technical Analysis of the Proposed Variants", IEEE Communications Standards Magazine, vol. 1, no. 4, Dec. 2017.

[9] M Centenaro, L Vangelista, A Zanella, M Zorzi, "Long-range communications in unlicensed bands: the rising stars in the IoT and smart city scenarios", IEEE Wireless Communications magazine. Vol. 23, pp. $60-67,2016$.

[10] "Handbook of Unmanned Aerial Vehicles", ISBN 978-90-481-9706-4, Springer 2015

[11] N. Neji, T. Mostfa and Y. Bestaoui, "Communication technology for Unmanned Aerial Vehicles: a qualitative assessment and application to Precision Agriculture", submitted to 2019 International Conference on Robotics and Automation (ICRA).

[12] Gianmarco Baldini,Member, Stan Karanasios, David Allen, and Fabrizio Vergari, "Survey of Wireless CommunicationTechnologies for Public Safety", IEEE Communications Surveys and Tutorials, Vol.16, No. 2, second quarter 2014. 